\title{
Identification of Dynamic Nonlinear Thermal Transfers for Precise Correction of Bias Induced by Temperature Variations
}

\author{
Céline Casenave • Gérard Montseny • Henri Camon • François Blard
}

Received: date / Accepted: date

\begin{abstract}
We present a general method for dynamic correction of biases induced by temperature variations. This method is simple to implement and useful when very high precision is required: it indeed allows significant improvements compared to standard static corrections. It is mainly based on a universal dynamic model allowing to describe macroscopic effects of complex underlying thermal phenomena inside the device. Some experimental results related to a MEMS with high precision pull-in voltage are presented in order to highlight the efficiency of the approach.
\end{abstract}

Keywords Temperature bias correction - Dynamic correction · Nonlinear correction · Dynamic thermal transfer · Diffusive representation · Least-square identification

\section{Introduction}

In general, high precision devices, such as MEMS devoted to metrology applications for example, need to include algorithms allowing to correct biases resulting from variations of the ambient temperature. Most of time, static corrections are involved, which means that the ambient temperature (measured by means of a sensor) is supposed to be also the temperature at any point of the system under consideration in its whole, in particular at any point of the MEMS device itself.

C. Casenave - G. Montseny - H. Camon - F. Blard

CNRS; LAAS; 7 avenue du colonel Roche, F-31077 Toulouse, France

Université de Toulouse; UPS, INSA, INP, ISAE; LAAS; F-31077

Toulouse, France

E-mail: casenave@laas.fr, montseny@laas.fr, camon@laas.fr, francois.blard@laas.fr
This is of course not exactly true because the ambient temperature is never strictly constant and so, due to the complex distributed nature of physical objects, the temperature inside the devices evolves following a complex diffusion equation. So, when measuring any physical quantity (for example a voltage), small residual fluctuations due to temperature variations remain in spite of the static correction, because dynamic thermal phenomena are involved, which cannot be exactly described by means of a static correspondence only.

In some cases, when very high precision is required, it can be judicious to envisage to build some dynamic corrections, elaborated from a suitable dynamic treatment of the temperature data, in such a way that the macroscopic effects resulting from the distributed evolution of the temperature inside the involved devices can be taken into account, and then corrected.

In this paper, we present a method devoted to such a dynamic correction. This method is mainly based on a universal dynamic model allowing to describe macroscopic effects of complex underlying thermal phenomena. It is simple to implement and useful when very high precision is required: it indeed allows significant improvements compared to standard static corrections.

The paper is organized as follows. In section 2 , we recall some notions about the standard method, that is the static correction. In section 3 , the principle of the proposed dynamic correction is presented in a general way. The presentation is mainly technical and formal, allowing concrete implementation of the proposed algorithms. In section 3, experimental results related to a MEMS with high precision pull-in voltage are presented and discussed. 


\section{Recalls on standard (static) polynomial correction}

Consider a quantity $U$ (to be measured), linked to the ambient temperature $T$ through a static relation of the form:

$U=f(T)$.

The aim is to correct the effect of the temperature $T$, that is to get an estimation (if possible optimal in some suitable sense) of the quantity $U_{0}=f\left(T_{0}\right)$, where a particular value $T_{0}$ is chosen a priori as the reference temperature.

\subsection{Static correction formulation}

If the function $f$ is regular enough (which is in general the case in concrete situations), $U$ can be expressed following the Taylor expansion around $T_{0}$ :

$U=f\left(T_{0}\right)+f^{\prime}\left(T_{0}\right)\left(T-T_{0}\right)+\frac{1}{2} f^{\prime \prime}\left(T_{0}\right)\left(T-T_{0}\right)^{2}+\ldots$,

that is, if we suppose that terms beyond the $n^{\text {th }}$ order can be neglected, we can consider the following static model of the quantity $U$ :

$U=U_{0}+\sum_{i=1}^{n} a_{i}\left(T-T_{0}\right)^{i}$.

So, if the coefficients $a_{i}$ have been previously accurately estimated (from experimental measurements) and $T$ is known, the bias induced by sufficiently slow temperature variations around $T_{0}$ can be corrected accordingly to the following relation deduced from (3):

$U_{0}=U-\sum_{i=1}^{n} a_{i}\left(T-T_{0}\right)^{i}$.

Indeed, when $U$ is measured $N$ times with some additive zero mean noise $w$, we then have $\tilde{U}_{k}=U_{k}+w_{k}$, $k=1: N$ and so, we get the correction relation:

$$
\begin{aligned}
\tilde{U}_{0, k} & =\tilde{U}_{k}-\sum_{i=1}^{n} a_{i}\left(T_{k}-T_{0}\right)^{i} \\
& =U_{k}-\sum_{i=1}^{n} a_{i}\left(T_{k}-T_{0}\right)^{i}+w_{k} \\
& =U_{0}+w_{k}, \quad k=1: N .
\end{aligned}
$$

An estimate of the unknown quantity $U_{0}$ can then be obtained by filtering the corrected data $\tilde{U}_{0 k}$, for example by:

$\hat{U}_{0}=\frac{1}{N} \sum_{k=1}^{N}\left(\tilde{U}_{k}-\sum_{i=1}^{n} a_{i}\left(T_{k}-T_{0}\right)^{i}\right)$.
We then have from (5) and thanks to the law of large numbers ( $\mathbb{E}$ designates the mathematical expectation):

$\mathbb{E}\left(\hat{U}_{0}\right)=U_{0}$,

with $\hat{U}_{0}=\frac{1}{N} \sum_{k=1}^{N}\left(U_{0}+w_{k}\right) \rightarrow U_{0}$ when $N \rightarrow \infty$,

the convergence being in the quadratic mean sense, that is $\mathbb{E}\left(\frac{1}{N} \sum_{k=1}^{N}\left(U_{0}+w_{k}\right)-U_{0}\right)^{2} \rightarrow 0$. So, the estimate $\hat{U}_{0}$ is convergent and unbiased: in that sense, the effects of temperature variations have been corrected.

Finally, the unknown quantity $U_{0}$ can be pursued (for example if $\tilde{U}$ is a voltage reference generated by a physical system) by means of the following moving average estimate based on the corrected data $\tilde{U}_{0, k}$ :

$\bar{U}_{0, k}=\frac{1}{N} \sum_{q=0}^{N-1}\left[\tilde{U}_{k-q}-\sum_{i=1}^{n} a_{i}\left(T_{k-q}-T_{0}\right)^{i}\right]$,

with the following residual estimation noise (inherited from the measurement noise):

$\varepsilon_{k}=\frac{1}{N} \sum_{q=0}^{N-1} w_{k-q}$.

\subsection{Preliminary estimation of the correction} coefficients from experimental data

The correction coefficients $a_{i}$ are estimated from experimental data, whose number must be sufficiently large to make the residual estimation error negligible. In practice, this step can be rather long, particularly if measurement data are significantly corrupted by noise. The classical estimation of coefficients $a_{i}$ from data $\tilde{U}_{k}, k=1: K$ is in general obtained from the classical least squares method, that is by solving the problem:

find $a \in \mathbb{R}^{n+1}$ such that:

$\sum_{k=1}^{K}\left(\tilde{U}_{k}-a_{0}-\sum_{i=1}^{n} a_{i}\left(T_{k}-T_{0}\right)^{i}\right)^{2}=\min$.

With $Q$ the matrix defined by:

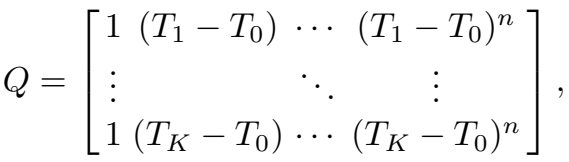

this problem can be rewritten under matrix form ${ }^{1}$ :

$\min _{a \in \mathbb{R}^{n+1}}(Q a-\tilde{U})^{T}(Q a-\tilde{U})$,

$1 X^{T}$ designating the transpose of the matrix $X$. 
the solution of which is classically given by:

$a=\left(Q^{T} Q+\varepsilon I\right)^{-1} Q^{T} \tilde{U}$

with $I$ the identity matrix and $\varepsilon \gtrsim 0$ a small parameter devoted to numerical conditioning of the matrix inversion.

Remark 1 Coefficient $a_{0}$ is simply the (optimal) estimate of the unknown quantity $U_{0}$ deduced from the set of measurement data $\left(T_{k}\right)_{1 \leqslant k \leqslant K}$ under consideration: due to measurement noise, it is not exactly equal to $U_{0}$.

\section{Precise correction of bias from identified dynamic thermal transfer}

3.1 Dynamic influence of the ambient temperature on a physical quantity

In practice, a physical system such as a MEMS with its environment is a complex distributed system: when the ambient temperature $T$ is not constant, the temperature of the system cannot be a simple scalar: it becomes a scalar field $\theta(t, x)$, where each (vector) value of the spatial variable $x$ is associated with a particular physical point of the system. If the variations of $T$ remain small, it can be reasonably supposed that the evolution of the field $\theta$ is governed by a complex but linear partial differential equation with input $T(t)$ of the abstract form ${ }^{2}$ :

$\frac{\partial \theta(t, x)}{\partial t}=A(\nabla) \theta(t, x)+B(x) T(t)$,

where $A(\nabla)$ is a second order linear differential operator (for example $A(\nabla)=\Delta$ for the classical heat equation in a given $3 \mathrm{D}$ domain), associated with suitable boundary conditions, and $B$ is the input operator, which describes how the exterior temperature $T$ acts on the evolution of $\theta$. Because equation (15) is in general defined on $t>t_{0}=0$, the initial condition must be added in order to make the problem complete:

$\theta(0, x)=\theta_{0}(x)$

Except in some ideal simple cases, such an equation is in practice very difficult or even impossible to formulate explicitly. Because temperature evolution is of diffusive nature, this equation however presents some specific features which will be used in the following section in order to built simple and efficient dynamic correction algorithms.

\footnotetext{
2 This abstract form will not be used by itself.
}

Similarly, a quantity $U$ relating to the physical system under consideration and depending at first sight on $T$ only, depends in general on the whole field $\theta$. As in the ideal static cases, even if the evolution of $\theta$ is governed by a linear dynamic model, this dependence can be nonlinear. For example, the quantity $U$ can depend nonlinearly on material dilatation, namely when devices with critical equilibrium states are involved, for example when pull-in behaviours are present (S. Krylov et al., 2008; M. Suhonen et al., 1998; A. Kärkkäinen et al., 2005; J. Kyynäräinen et al., 2001; C. Casenave et al., 2010).

We can then write (formally):

$U=\mathbf{F}(\theta)$,

where $\mathbf{F}$ is a spatial operator. Under this form, the quantity $U$ explicitly appears as an output of (15) and the dynamic (in general nonlinear) transfer $T \longmapsto U$ is then resulting from the input-output correspondence defined by $(15,17)$. This is the starting point of the approach.

In most of cases, the evolution of $T(t)$ is slow enough, in such a way that and it can be considered that the difference $|\theta(t, x)-T(t)|$ remains negligible and then, the static approach previously described is sufficient to get accurate corrections. In some cases however, namely when very high precision is required, small differences between $\theta(t, x)$ and $T(t)$, which result from the dynamic and distributed nature of model (15), can generate some significant residual biases which cannot be suppressed by static correction. In such cases, the static model (3) is no longer sufficiently accurate and a dynamic correction is needed.

3.2 Dynamic correction of bias resulting from temperature variations

The correction method described here-after is mainly based on an explicit and universal differential inputoutput model of the dynamic transfer $(15,17)$. This model is closely related to the so-called diffusive representation: more details about this theory and its applications in identification of nonlinear complex models or other various fields can be found for example in (C. Casenave and G. Montseny, 2010; G. Montseny, 2005; C. Casenave, 2009).

\subsubsection{Exact theoretical formulation}

First, thanks to the linear and diffusive nature of equation (15), a generic 1D diffusive input-state equation is considered in order to generate a family of terms $\psi(t, \xi)$, each one associated with a pulsation $\xi$, and which will 
be able to describe in some sense the underlying thermal dynamics. By assuming for simplicity that the mean ambient temperature is

$T_{0}=0$,

this model is:

$\left\{\begin{array}{l}\frac{\partial \psi(t, \xi)}{\partial t}=-\xi \psi(t, \xi)+T(t), \xi>0, t>0 \\ \psi(0, \xi)=0\end{array}\right.$

On the other hand, from linearity, the contribution of the initial condition $\theta_{0}$ is separately expressed from the following exponential family associated with a family of terms $\psi_{0}(\xi)$ :

$\psi_{0}(t, \xi)=e^{-\xi t} \psi_{0}(\xi)$

It can be noted that the function $(t, \xi) \mapsto \psi(t, \xi)+$ $\psi_{0}(t, \xi)$ is solution of (18) with initial condition $\psi_{0}(\xi)$ in place of 0 .

We then define the function:

$\Psi(t, \xi):=\psi(t, \xi)+\psi_{0}(t, \xi)-T(t)$

which expresses the difference between the instantaneous ambient temperature $T(t)$ and the field $\psi(t, \xi)+$ $\psi_{0}(t, \xi)$. It is that function which will be devoted to dynamic correction.

Then, it can be shown that under very weak hypothesis, the unknown dynamic transfer $T \mapsto U$ can be expressed under the form $U=G(T, \Psi)$ with $G$ a suitable nonlinear operator with generic formulation:

$$
\begin{aligned}
& U(t)=U_{0}+\sum_{i \geqslant 0} a_{i} T(t)^{i} \\
& +\int \mu_{1}(\xi) \Psi(t, \xi) d \xi \\
& +\iint \mu_{2}\left(\xi_{1}, \xi_{2}\right) \Psi\left(t, \xi_{1}\right) \Psi\left(t, \xi_{2}\right) d \xi_{1} d \xi_{2} \\
& +\iiint \mu_{3}\left(\xi_{1}, \xi_{2}, \xi_{3}\right) \Psi\left(t, \xi_{1}\right) \Psi\left(t, \xi_{2}\right) \Psi\left(t, \xi_{3}\right) d \xi_{1} d \xi_{2} d \xi_{3} \\
& +\iiint \int \ldots
\end{aligned}
$$

in other words, $U(t)$ is synthesized from the ambient temperature $T(t)$ and the previously defined field $\Psi(t, \xi)$, via a family of "parameters" $a_{i}$ and $\mu_{i}$. This expression involves a new family of fields directly deduced from $\Psi(t, \xi)$ and which are denoted:

$\Psi^{\otimes i}(t, \xi):=\Psi\left(t, \xi_{1}\right) \Psi\left(t, \xi_{2}\right) \ldots \Psi\left(t, \xi_{i}\right)$
Remark 2 In some sense, each of these fields is seen as an elementary (nonlinear) dynamic component possibly generated by the underlying thermal transfer $T \mapsto \theta$. Note however that the fields $\Psi^{\otimes i}$ have no direct physical interpretation; from the mathematical point of view, these fields are simply functional analogous of the quantities $T^{i}$ involved in (2): they relate to the Taylor expansion based on the "operatorial" formulation (17) (rather the standard "functional" one (1)).

Finally, by use of the convenient scalar product notation:

$\langle f, g\rangle_{i}=\int \cdots \int_{\mathbb{R}^{i}} f(\boldsymbol{\xi}) g(\boldsymbol{\xi}) d \boldsymbol{\xi}$

(21) can be rewritten under the compact and quite simple form (we have omitted the $t$ variable for simplicity):

$U=U_{0}+\sum_{i \geqslant 0} a_{i} T^{i}+\sum_{i \geqslant 0}\left\langle\mu_{i}, \Psi^{\otimes i}\right\rangle_{i}$

with $\Psi$ deduced from $T$ via the dynamic relations $(18,19,20)$ (involving "initial condition coefficients" $\psi_{0}$ ); in the above expression, the static and dynamic parts are respectively $\sum_{i} a_{i} T^{i}$ and $\sum_{i}\left\langle\mu_{i}, \Psi^{\otimes i}\right\rangle_{i}$, associated with respective "coefficients" $a_{i}$ and $\mu_{i}$.

\subsubsection{Numerical formulation}

Now recall that $\Psi(t,$.$) and \mu_{i}$ are real functions defined on the respective variables $\xi \in \mathbb{R}$ and $\xi_{i}=\left(\xi_{1}, \xi_{2}, \ldots, \xi_{i}\right) \in$ $\mathbb{R}^{i}$; so, to get implementable algorithms, a numerical approximation of the dynamic model of $U$ previously introduced must be derived in order to get only a finite number of involved real coefficients (G. Montseny, 2005).

Because equation (18) is a diagonal system of ordinary first order differential equations, an efficient approximation of its solution $\psi(t, \xi)$ is deduced simply by considering a finite number of terms $\psi_{j}(t):=\psi\left(t, \xi_{j}\right)$, with $\left\{\xi_{j}\right\}$ covering the band of useful frequencies associated with the dynamic transfer $T \rightarrow U$. Similarly, the associated approximation of $\Psi(t, \xi)$ is deduced by taking:

$\Psi_{j}(t):=\Psi\left(t, \xi_{j}\right)=\psi\left(t, \xi_{j}\right)+\psi_{0}\left(t, \xi_{j}\right)-T(t)$, 
and so, a discrete equivalent of (21) is gotten under the form:

$$
\begin{aligned}
& U(t)=U_{0}+\sum_{i=1}^{n_{\mathrm{s}}} a_{i} T(t)^{i} \\
& +\sum_{j^{\prime}} \mu_{1}^{j^{\prime}} \Psi_{j^{\prime}}(t) \\
& +\sum_{j^{\prime}, j^{\prime \prime}} \mu_{2}^{j^{\prime} j^{\prime \prime}} \Psi_{j^{\prime}}(t) \Psi_{j^{\prime \prime}}(t) \\
& +\ldots \\
& +\sum_{j^{\prime}, j^{\prime \prime}, \ldots, j^{\left(n_{\mathrm{d}}\right)}} \mu_{n_{\mathrm{d}}}^{j^{\prime} j^{\prime \prime} \ldots j^{\left(n_{\mathrm{d}}\right)}} \Psi_{j^{\prime}}(t) \Psi_{j^{\prime \prime}}(t) \ldots \Psi_{j^{\left(n_{\mathrm{d}}\right)}}(t) .
\end{aligned}
$$

By use of the notations:

$$
\begin{aligned}
\mu_{i} & :=\left(\mu_{i}^{j^{\prime} j^{\prime \prime} \ldots j^{(i)}}\right), \\
\boldsymbol{\Psi} & :=\left(\Psi_{j}\right), \quad \Psi^{\otimes i}:=\left(\Psi_{j^{\prime}} \Psi_{j^{\prime \prime} \ldots \Psi_{j^{(i)}}}\right), \\
\mu_{i} \cdot \boldsymbol{\Phi} & :=\sum_{j^{\prime}, j^{\prime \prime}, \ldots j^{(i)}} \mu_{i}^{j^{\prime} j^{\prime \prime} \ldots j^{(i)}} \Phi_{j^{\prime} j^{\prime \prime} \ldots j^{(i)}},
\end{aligned}
$$

and omitting the $t$ variable for simplicity, we get the quite simple and expressive equivalent formulation, well adapted to modern softwares devoted to numerical computing:

$U=U_{0}+\sum_{i=1}^{n_{\mathrm{s}}} a_{i} T^{i}+\sum_{i=1}^{n_{\mathrm{d}}} \mu_{i} \cdot \mathbf{\Psi}^{\otimes i}$

with $\Psi_{j}(t)=\psi\left(t, \xi_{j}\right)+\psi_{0}\left(t, \xi_{j}\right)-T(t)$ and $\psi, \psi_{0}$ given by $(18,19)$; the $\mu_{i}$ and $\boldsymbol{\Psi}^{\otimes i}$ are real valued tensors of rank $i$, with dimension ${ }^{3} J_{i}^{\prime} \times J_{i}^{\prime \prime} \times \ldots \times J_{i}^{(i)}$ to be chosen, as usual, to realize the best compromise between accuracy and numerical cost. Note that clearly, as in the static case (3), all the (tensor) coefficients $a_{i}$ and $\mu_{i}$ of this model are linearly involved.

Remark 3 In most of practical situations, thanks to the rapid convergence of the series (25) when $n_{\mathrm{s}}, J_{i}^{(k)} \rightarrow$ $+\infty$, only a small number of tensor coefficients $\mu_{i}^{j^{\prime} j^{\prime \prime} \ldots j^{(i)}}$ be sufficient to get very accurate approximations of the dynamic nonlinear transfer $T \mapsto U$. Such questions will be studied in a further paper devoted to mathematical and numerical analysis.

In particular, if the variations of $T(t)$ are small enough, the contributions of the nonlinear terms $\Psi_{j^{\prime}} \Psi_{j^{\prime \prime}}, \Psi_{j^{\prime}} \Psi_{j^{\prime \prime}} \Psi_{j}$ etc., can be neglected and the following simplified synthesis with $n_{\mathrm{d}}=1$ (that is linear in the dynamic part)

3 That is the number of coefficients; in the particular case $J_{i}^{(k)}=J$ for any $i, k$, this dimension is simply $J^{i}$. can be yet sufficient to get significant improvement compared to the static model (3):

$$
\begin{aligned}
U(t)= & U_{0}+\sum_{i=1}^{n_{\mathrm{s}}} a_{i} T(t)^{i} \\
& +\sum_{j=1}^{J} \mu_{j} \Psi_{j}(t),
\end{aligned}
$$

in such a way that (26) can be rewritten, from (19) and with $\nu_{j}:=\mu_{j} \psi_{0}\left(\xi_{j}\right)$, under the following form in which all the parameters (including the "initial condition" ones), that is $a_{i}, \mu_{j}, \nu_{j}$, are linearly involved:

$$
\begin{aligned}
U(t)= & U_{0}+\sum_{i=1}^{n_{\mathrm{s}}} a_{i} T(t)^{i} \\
& +\sum_{j=1}^{J} \mu_{j}\left(\psi\left(t, \xi_{j}\right)-T(t)\right) \\
& +\sum_{j=1}^{J} \nu_{j} e^{-\xi_{j} t}
\end{aligned}
$$

\subsubsection{Algorithm for dynamic correction}

Similarly to the static case, when $U$ is measured at times $t_{k}=k \Delta t$ with some additive zero mean noise $w_{k}$, that is $\tilde{U}_{k}=U_{k}+w_{k}$, and because $e^{-\xi_{j} t_{k}} \rightarrow 0$ when $k \rightarrow+\infty$, we finally get the following asymptotic dynamic correction formula deduced from (25), suitable for $k$ large enough (i.e. is in such a way that the quantities $e^{-\xi_{j} t_{k}}$ are quite negligible):

$$
\tilde{U}_{0, k}=\tilde{U}_{k}-\sum_{i=1}^{n_{\mathrm{s}}} a_{i}\left(T_{k}\right)^{i}-\sum_{i=1}^{n_{\mathrm{d}}} \mu_{i} \cdot \overline{\mathbf{\Psi}}_{k}^{\otimes i}
$$

where the vectors

$\overline{\mathbf{\Psi}}_{k}:=\left(\Psi_{j, k}-\psi_{0}\left(\xi_{j}\right) e^{-\xi_{j} t_{k}}\right)=\left(\psi_{j, k}-T_{k}\right)$,

on which is based the dynamic correction, are computed from the temperature data $T_{k}:=T\left(t_{k}\right)$ via the following discrete-time dynamic relation deduced from inteillgration of (18):

$$
\psi_{j, k+1}=e^{-\xi_{j} \Delta t} \psi_{j, k}+\frac{1-e^{-\xi_{j} \Delta t}}{\xi_{j}} T_{k}, \quad \psi_{j, 0}=0 .
$$

In the simplified case $n_{\mathrm{d}}=1$ (that is the correc,tion is linear with respect to the dynamic part), (28) becomes:

$$
\tilde{U}_{0, k}=\tilde{U}_{k}-\sum_{i=1}^{n_{\mathrm{s}}} a_{i}\left(T_{k}\right)^{i}-\sum_{j=1}^{J} \mu_{j}\left(\psi_{j, k}-T_{k}\right) .
$$


Exactly as in the static case, $\tilde{U}_{0, k}=U_{0}+w_{k}$ and so, estimates $\hat{U}_{0}$ or $\bar{U}_{0, k}$ of the quantity $U_{0}$ can yet be taken as:

$\hat{U}_{0}=\frac{1}{N} \sum_{k=1}^{N} \tilde{U}_{0, k}, \quad \bar{U}_{0, k}=\frac{1}{N} \sum_{q=0}^{N-1} \tilde{U}_{0, k-q}$

and we have yet, from (28) and the law of large numbers:

$\mathbb{E}\left(\hat{U}_{0}\right)=U_{0}$

$\hat{U}_{0}=\frac{1}{N} \sum_{k=1}^{N}\left(U_{0}+w_{k}\right) \rightarrow U_{0}$ when $N \rightarrow \infty$.

Finally, the unknown quantity $U_{0}$ can be pursued by means of the following moving average estimate:

$\bar{U}_{0, k}=\frac{1}{N} \sum_{q=0}^{N-1}\left[\tilde{U}_{k-q}-\sum_{i=1}^{n} a_{i}\left(T_{k-q}\right)^{i}-\sum_{i=1}^{n_{\mathrm{d}}} \mu_{i} \cdot \overline{\mathbf{\Psi}}_{k}^{\otimes i}\right]$,

with the following residual estimation noise:

$\varepsilon_{k}=\frac{1}{N} \sum_{q=0}^{N-1} w_{k-q}$

\subsubsection{On the practical choice of the pulsations $\xi_{j}$}

As stated in section 3.2.2, for concrete implementation of dynamic corrections, the first (and essential) step is to correctly chose the pulsations $\xi_{j}, j=1: J$. The number $J$ must of course be as small as possible. From (18), it clearly appears that $\Psi_{j}(t)=\Psi\left(t, \xi_{j}\right)$ results from simply filtering $T(t)$ by a low-pass filter with cut-off frequency $f_{i}=\frac{\xi_{j}}{2 \pi}$. By assuming that $\xi_{1}=\xi_{\min }<\xi_{2}<$ $\ldots<\xi_{J}=\xi_{\max }$, the frequency band covered by the set of $f_{i}$ (that is $\left[f_{1}, f_{J}\right]$ ) as well as the number $J$ is empirically chosen from analysis of the behaviour of the residual estimation noise (36).

A simple method consists in implementing, on a given set of stored data $\left\{\tilde{U}_{k}, T_{k}\right\}$, an iterative process initiated with the standard static correction. The behaviour of the residual estimation noise then suggests to introduce some $\xi_{j}$ and the linear dynamic correction based on these $\xi_{j}$ is implemented, etc. At each step, the variance of the residual noise must decrease; otherwise the process is ended.
3.3 Preliminary estimation of the correction coefficients from experimental data

The correction tensors $a_{i}, \mu_{i}$ are to be firstly estimated from experimental data whose number must be sufficient to get negligible estimation errors. This number is all the larger as the number of scalar coefficient to be identified is large. Furthermore, because it is desirable that contributions of possible non null initial conditions are negligible (in order to avoid biases induced by such contributions), data are to be stored only after a sufficiently long time $t_{k_{0}}=k_{0} \Delta t$. This leads in general to very long times for data acquisition and for this reason, the dynamic model of $U$ must be chosen with as few coefficients as possible, in particular in the dynamic part which involves tensors, whose dimension increases very quickly.

An optimal estimation from (noised) measured data $\tilde{U}_{k}, k=k_{0}: K$ is obtained again by means of a least square method, for example by minimizing (with respect to the scalar parameters $\left.a_{i}, \mu_{i}^{j^{\prime} j^{\prime \prime} \ldots j^{(i)}} \in \mathbb{R}\right)$ the quantity:

$\mathcal{J}=\sum_{k=k_{0}}^{K}\left(\tilde{U}_{k}-a_{0}-\sum_{i=1}^{n_{\mathrm{s}}} a_{i}\left(T_{k}\right)^{i}-\sum_{i=1}^{n_{\mathrm{d}}} \mu_{i} \cdot \overline{\mathbf{\Psi}}\left(T_{k}\right)^{\otimes i}\right)^{2} ;$

this problem can be rewritten under matrix form ${ }^{4}$, with $\left(a, \mu^{*}\right)^{T}$ the column vector with terms all the scalar coefficients $a_{i}, \mu_{i}^{j^{\prime} j^{\prime \prime} \ldots j^{(i)}}$ :

$\min _{a, \mu^{*}}\left[\mathbf{Q} \cdot\left(\begin{array}{c}a \\ \mu^{*}\end{array}\right)-\tilde{U}\right]^{T} \cdot\left[\mathbf{Q} \cdot\left(\begin{array}{c}a \\ \mu^{*}\end{array}\right)-\tilde{U}\right]$

the solution is then classically given by:

$\left(\begin{array}{c}a \\ \mu^{*}\end{array}\right)=\left[\mathbf{Q}^{T} \mathbf{Q}+\varepsilon \mathbf{I}\right]^{-1} \mathbf{Q}^{T} \tilde{U}$.

In the particular case $n_{\mathrm{d}}=1$, it results from $(27)$ that the "initial condition parameters" $\nu_{j}=\mu_{j} \psi_{0}\left(\xi_{j}\right)$ can easily be identified together with $a_{i}$ and $\mu_{j}$, which allows to avoid undesirable biases on $a_{i}$ and $\mu_{j}$ even if the data are stored from the beginning of the measurement process. In this case, the quantity to be minimized becomes:

$$
\begin{aligned}
\mathcal{J}=\sum_{k=1}^{K} & \left(\tilde{U}_{k}-a_{0}-\sum_{i=1}^{n_{\mathrm{s}}} a_{i}\left(T_{k}\right)^{i}\right. \\
& \left.-\sum_{j=1}^{J} \mu_{j}\left(\psi_{j, k}-T_{k}\right)-\sum_{j=1}^{J} \nu_{j} e^{-\xi t_{k}}\right)^{2}
\end{aligned}
$$

4 The terms of the matrix $\mathbf{Q}$ are constituted by the ones of $\left(T_{k}\right)^{i}$ and $\bar{\Psi}\left(T_{k}\right)^{\otimes i}$. 
this problem is rewritten under matrix form with $(a, \mu, \nu)^{T}$ $\in \mathbb{R}^{(n+1)} \times \mathbb{R}^{J} \times \mathbb{R}^{J}$ :

$\min _{a, \mu, \nu}\left[\mathbf{Q} \cdot\left(\begin{array}{c}a \\ \mu \\ \nu\end{array}\right)-\tilde{U}\right]^{T} \cdot\left[\mathbf{Q} \cdot\left(\begin{array}{c}a \\ \mu \\ \nu\end{array}\right)-\tilde{U}\right]$,

the solution of which is given by:

$$
\left(\begin{array}{c}
a \\
\mu \\
\nu
\end{array}\right)=\left[\mathbf{Q}^{T} \mathbf{Q}+\varepsilon \mathbf{I}\right]^{-1} \mathbf{Q}^{T} \tilde{U}
$$

\section{Application to a high precision voltage reference based on an electrostatically actuated MEMS}

In the sequel, we present some experimental results obtained by use of the method described above. The physical system under consideration is an electrostatically actuated MEMS devoted to the construction of a voltage reference of high precision and stability. From the electrical point of view, it is simply a variable capacitance in which the electrostatic force is opposed to the one of a mechanical spring between the two electrodes. So, there exists a so-called pull-in voltage, beyond which the mechanical force is no more able to balance the electrostatic one: this defines the reference voltage, which is determined by the only mechanical design of the MEMS (M. Suhonen et al., 1998; A. Kärkkäinen et al., 2005; J. Kyynäräinen et al., 2001).

For stability tests, the electrical environment of the MEMS is shown (in a simplified form) in Fig. 2. The DC voltage applied between the MEMS electrodes is chosen slightly less than the pull-in voltage. Variations of the ambient temperature generate variations of the electrical capacitance of the $\mathrm{MEMS}^{5}$ and therefore variations of the measured AC voltage.

4.1 A preliminary analysis of thermal dynamics and their consequences

To get a low pull-in voltage (about 10V), the MEMS under consideration needs to satisfy some specific features, namely a large mobile Silicon electrode with complex design, in order to get a great electrical capacitance, suspended by 4 very thin Silicon springs, which allows to get a low stiffness (see Fig. 1). This particular geometrical situation suggests that thermal transfers to the mobile electrode will be significantly slowed due to the high thermal resistance of the suspension springs.

\footnotetext{
${ }^{5}$ NB: this capacitance is around $10 \mathrm{pF}$.
}

By considering that the mobile electrode has a thermal capacitance $C$ and the springs has an equivalent thermal resistance $R$, we can perform a simplified analysis of the resulting characteristic time constant and of its consequences according to variations of the ambient temperature.

First, the dimensions of the springs are $265 \mu \mathrm{m} \times$ $4 \mu \mathrm{m} \times 60 \mu \mathrm{m}$, that gives a resulting thermal resistance about $R=7.5 \times 10^{3} \mathrm{~K} / \mathrm{W}$. Second, the dimensions of the mobile electrode are about $2000 \mu \mathrm{m} \times 150 \mu \mathrm{m} \times 60 \mu \mathrm{m}$, which leads to a mass about $40 \mu \mathrm{g}$; so, its thermal capacitance is about $C=4 \times 10^{-5} \mathrm{~J} / \mathrm{K}$. Consequently, the time constant of the associated thermal transfer is about $\tau=R C=2 \times 10^{3} \times 4 \times 10^{-5} \simeq 10^{-1}$ s.

By denoting $T_{\mathrm{m}}$ the temperature of the mobile electrode (supposed to be uniform for simplicity) and $T$ the temperature of the substrate (also supposed to be uniform and equal to the ambient temperature), we therefore get the following differential equation:

$\frac{d T_{\mathrm{m}}}{d t}=\frac{1}{\tau}\left(T-T_{\mathrm{m}}\right)$

Consequently, if the ambient temperature variation $\frac{d T}{d t}$ is supposed to be constant in some time interval much larger than $0.1 \mathrm{~s}$, then we can consider that in this time interval, $\frac{d T_{\mathrm{m}}}{d t}=\frac{d T}{d t}$ and so the difference $\Delta T:=T-T_{\mathrm{m}}$ is constant and equal to:

$\Delta T=\tau \frac{d T}{d t}$

As an example, with $\left|\frac{d T}{d t}\right|=10^{-3} \mathrm{~K} / \mathrm{s}$, we have $|\Delta T|=$ $10^{-4} \mathrm{~K}$ : in most of situations, this difference is quite negligible. However, recall that our ultimate goal is to build high quality voltage references based on such MEMS, with expected stability about 1 ppm; taking into account that for the device under consideration, the coefficient $\sigma:=\frac{\Delta U}{\Delta T}$ is about $\sigma=4 \times 10^{-3} \mathrm{~V} / \mathrm{K}$ (cf. Fig. 3,7), then, the resulting relative variation of the measured voltage should be about $\frac{|\Delta U|}{U}=\frac{|\sigma \Delta T|}{U} \simeq$ $\frac{4 \times 10^{-3} \times 10^{-4}}{0.1}=4 \mathrm{ppm}$, which is quite excessive according to the expected precision.

In addition, some biases resulting from such dynamic components not taken into account in the identification process could in practice generate erroneous estimations of the correction coefficients. So, given an expected relative precision $\varepsilon$, the static correction can be considered as quite inappropriate if:

$\frac{|\sigma| \tau}{U}\left|\frac{d T}{d t}\right|>\varepsilon$

In the present case (i.e. $\varepsilon=10^{-6}$ ), we can conclude that dynamic correction is needed if $\left|\frac{d T}{d t}\right|$ can exceed $2 \times$ 
$10^{-4} \mathrm{~K} / \mathrm{s} \simeq 1 \mathrm{~K} / \mathrm{h}$. Such ambient temperature variations are frequent in standard environments.

Finally, note that beyond the MEMS itself, other devices (including the temperature sensor and the whole measurement equipment) possibly involve thermal time constants that cannot be neglected. Due to the large size of some of these devices, time constants about 1s or even much more cannot be excluded: in such a case, the maximal admissible $\left|\frac{d T}{d t}\right|$, beyond which static correction is no longer appropriate, is less than $0.1 \mathrm{~K} / \mathrm{h}$ : such a constraint is widely incompatible with the standard environments in which the measurements have been made and so, we can expect that a dynamic correction should bring significant improvements versus the static one. This will be confirmed in the next section where the dynamic correction presented above is implemented on real data.

\subsection{Experimental results}

The measured AC voltage is shown in Fig. 3; the sample period is $\Delta t=15 \mathrm{~s}$. Significant variations visible: they mainly result from variations of the ambient temperature $T(t)$, given in Fig. 7. For static correction, we have taken $n=1$ (linear correction) or $n=2$. For dynamic correction, $J=6$ time constants $1 / \xi_{j}$ have been used.

In Fig. 4, we can see both the measured data and a dynamic correction of the form:

$a_{0}-\sum_{i=1}^{2} a_{i}\left(T_{k}\right)^{i}-\sum_{j=1}^{6} \mu_{j}\left(\psi_{j, k}-T_{k}\right)$

(where $a_{0}$ is the estimate of the unknown AC voltage $\left.U_{0}\right)$.

The correction is then subtracted to the measured data to get the so-called residual voltage variations. These residual variations are shown in Fig. 5, 6, either non smoothed, or smoothed by use of a standard moving average filter. Clearly, a significant improvement is gotten when dynamic correction is implemented.

Evolutions of the temperature $T$ and the "auxiliary temperatures" $\psi_{j}+\psi_{0, j}$ are shown in Fig. 7,8,9. In Fig. 10, we can see the contribution to these evolutions of the terms inherited from initial conditions, that is: $\psi_{0, j}\left(t_{k}\right)=\frac{\nu_{j}}{\mu_{j}} e^{-\xi_{j} t_{k}}$ (recall that, as mentioned in section 3.2.2, the so-identified initial conditions are $\left.\psi_{0, j}(0)=\frac{\nu_{j}}{\mu_{j}}\right)$.

Finally, Fig. 11 shows the differences between the auxiliary temperatures and $T$, that is the functions $\Psi_{j}=$ $\psi_{j}+\psi_{0, j}-T$.

From a more quantitative point of view, we have:

\begin{tabular}{|l|c|c|}
\cline { 2 - 3 } \multicolumn{1}{c|}{} & non smoothed & smoothed \\
\hline stat. lin. & $9.985 \times 10^{-5}$ & $4.911 \times 10^{-5}$ \\
stat. nonlin. & $9.780 \times 10^{-5}$ & $4.471 \times 10^{-5}$ \\
dyn. lin. & $6.769 \times 10^{-5}$ & $1.331 \times 10^{-5}$ \\
dyn. nonlin. & $6.725 \times 10^{-5}$ & $1.173 \times 10^{-5}$ \\
\hline
\end{tabular}

Table 1 Standard deviation of the AC voltage after correction $(\mathrm{mV})$.

\begin{tabular}{|l|l|}
\hline stat. lin. & $-27,3 \mathrm{~dB}$ \\
stat. nonlin. & $-27.9 \mathrm{~dB}$ \\
dyn. lin. & $-38.6 \mathrm{~dB}$ \\
dyn. nonlin. & $-39.5 \mathrm{~dB}$ \\
\hline
\end{tabular}

Table 2 Reduction of the residual variations of the AC voltage

\begin{tabular}{|l|l|}
\hline linear & $-11.3 \mathrm{~dB}$ \\
nonlinear & $-11.6 \mathrm{~dB}$ \\
\hline
\end{tabular}

Table 3 Improvement by use of dynamic correction versus static one.

- mean measured AC voltage: $0.1040 \mathrm{mV}$;

- standard deviation before correction of the AC voltage: $1.114 \times 10^{-3} \mathrm{mV}$;

- results obtained with static and dynamic corrections given in tables 1, 2 and 3.

Remark 4 Once the correction coefficients have been identified from the process described in section 3.3, the real-time correction algorithms are given by (5) for the static case and by $(30,31)$ for the dynamic case. By considering the only linear correction for simplicity, these algorithm are of the respective forms (N.B. recall that the reference temperature $T_{0}$ is taken equal to 0 ):

$\tilde{U}_{0, k}=\tilde{U}_{k}-a_{1} T_{k}$,

$\left\{\begin{array}{l}\psi_{j, k+1}=\alpha \psi_{j, k}+\beta T_{k}, \quad j=1: 6 \\ \tilde{U}_{0, k}=\tilde{U}_{k}-a_{1} T_{k}-\sum_{j=1}^{6} \mu_{j}\left(\psi_{j, k}-T_{k}\right),\end{array}\right.$

and it is easy to deduce the respective numerical costs:

2 ops $/ \Delta t$ for static correction,

$6 \times 6+2=38 \mathrm{ops} / \Delta t$ for dynamic correction.

So, although the real time numerical cost of dynamic correction is much greater, it remains negligible in general. 


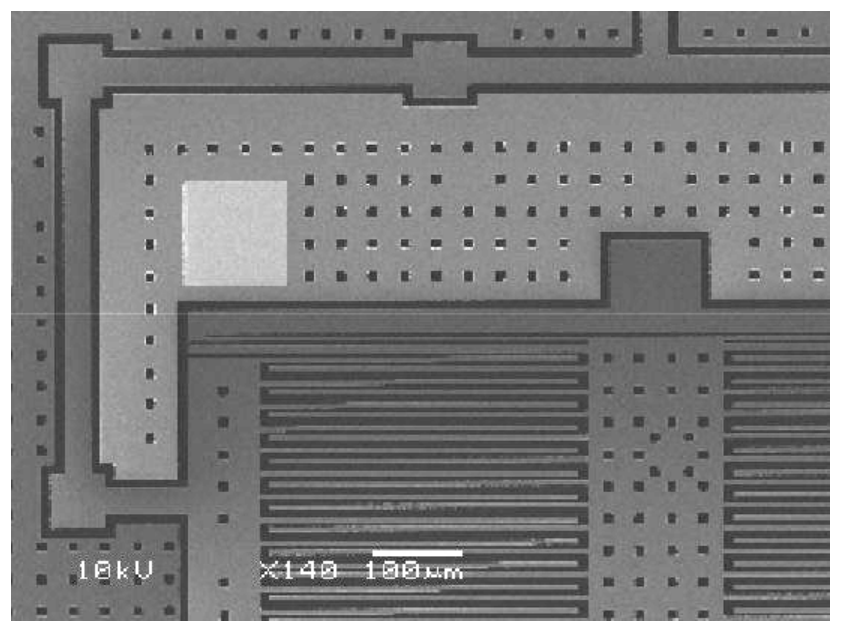

Fig. 1 The electrostatically actuated MEMS under consideration

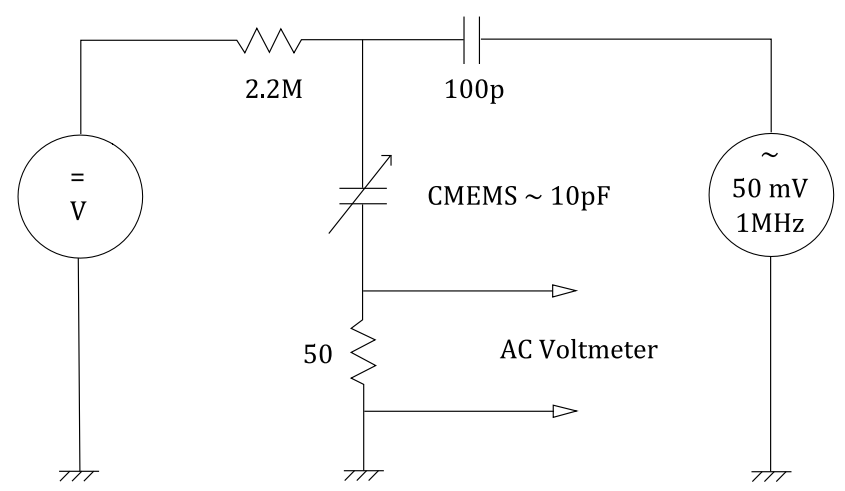

Fig. 2 Electrical environment of the MEMS for data acquisition

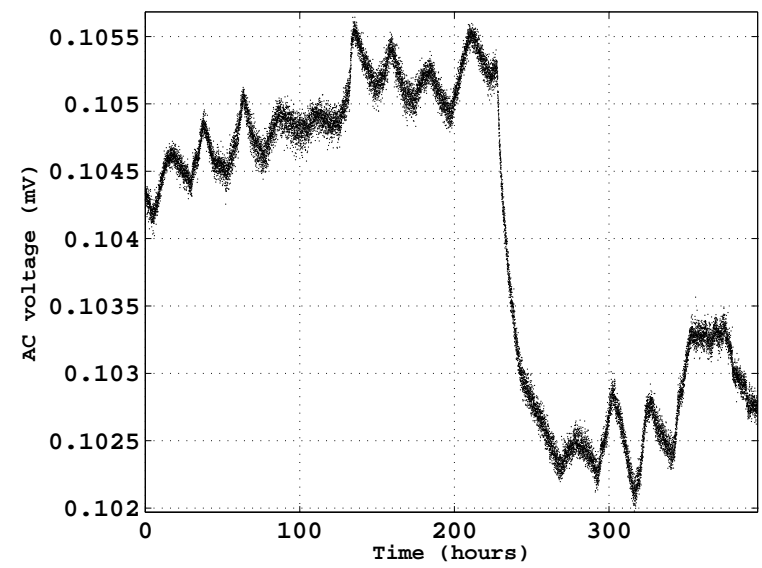

Fig. 3 Measured AC voltage

\section{Conclusion}

As clearly highlighted by the quantitative results presented above, dynamic correction can in some cases generate significant improvements. However, in the present results, some residual variations remain after correc-

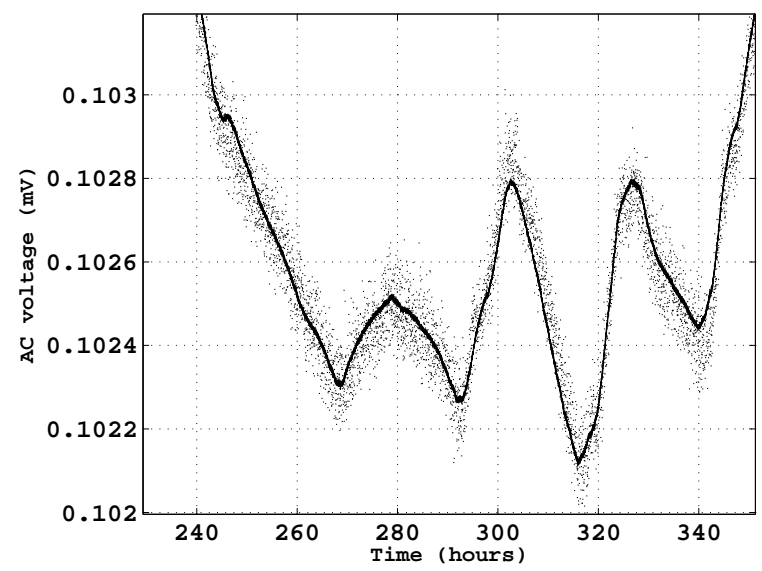

Fig. 4 Measured AC voltage (:), dynamic correction (-)
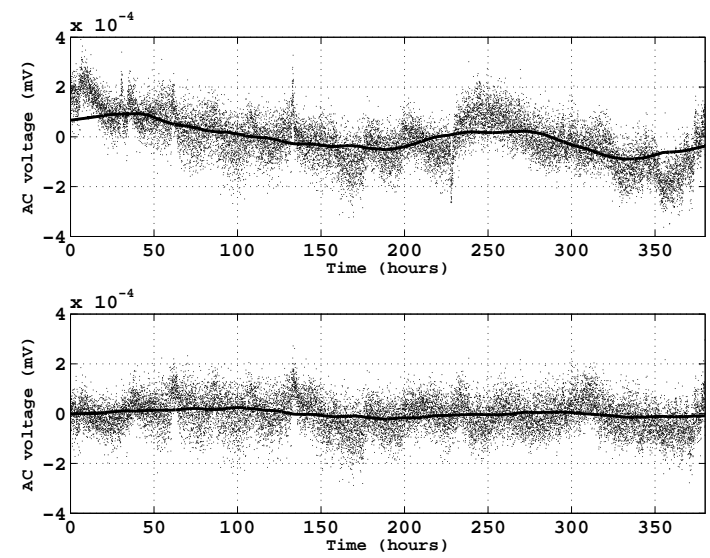

Fig. 5 Residual variations: with static correction (top), with dynamic correction (bottom); non smoothed (:), smoothed (-)

tion; it is probable that they are not entirely induced by noises only: indeed, great deviations of $\Psi_{j}(t)$ sometimes appear (see Fig. 11), which suggests that the only linear part of the dynamic correction is no longer sufficient in such cases. So, it will be judicious to involve higher order (nonlinear) terms of the series (24), in particular quadratic ones: $\mu_{2}^{j^{\prime} j^{\prime \prime}} \Psi_{j^{\prime}}(t) \Psi_{j^{\prime \prime}}(t)$.

This will be studied in a further work, with a new set of data devoted specifically to this problem. As stated in section 3.3, this new set of data will be much larger, first because more coefficients are to be identified, but especially because the data will be stored only after a long transient phase during which possible non null (and unknown!) temperature initial conditions inside the device can generate some excessive estimation biases.

From the theoretical point of view, the problem of estimating such initial conditions when the order $n_{\mathrm{d}}$ of 


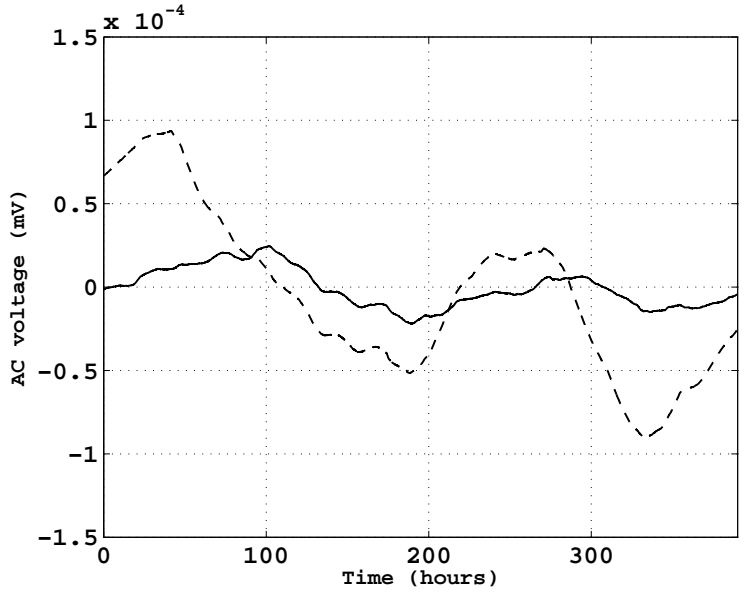

Fig. 6 Smoothed residual variations: static correction (- -), dynamic correction $(-)$

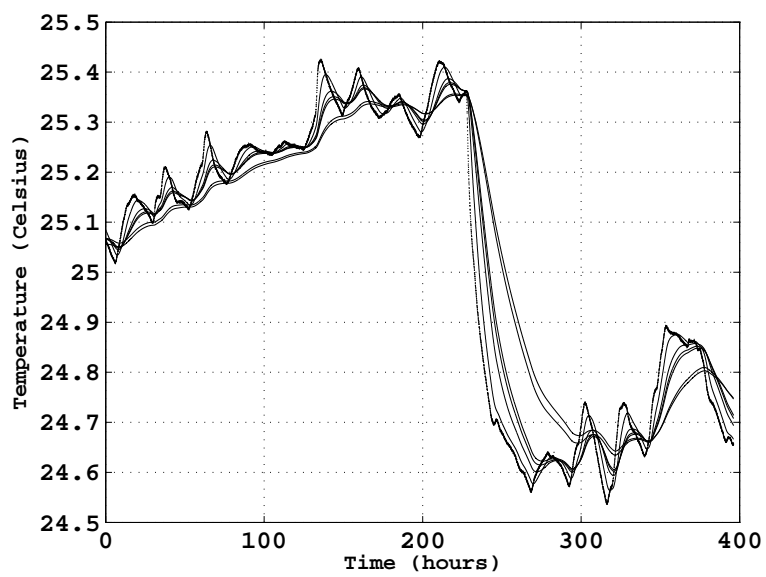

Fig. 7 Temperatures: $T(t)$ (bold) and $\psi_{j}(t)+\psi_{0, j}(t)$.

the dynamic correction is greater than 1 is also currently under study.

\section{References}

H. Camon, C. Ganibal, N. Raphoz, M. Trzmiel, C. Pisella, C. Martinez, S. Valette, Solving functional reliability issue for an optical electrostatic switch, Microsystem Technologies, Vol. 14, N. 7, July 2008.

H. Camon, F. Larnaudie, Fabrication, simulation and experiment of a rotating electrostatic silicon mirror with large angular deflection, 13th Int. Micro Electro Mechanical Systems (MEMS 2000), Miyazaki (Japan), pp. 645-650, Jan. 23-27, 2000.

C. Casenave, Représentation diffusive et inversion opératorielle pour l'analyse et la résolution de problèmes dynamiques non locaux, $\mathrm{PhD}$ thesis, Toulouse (France), déc. 2009.

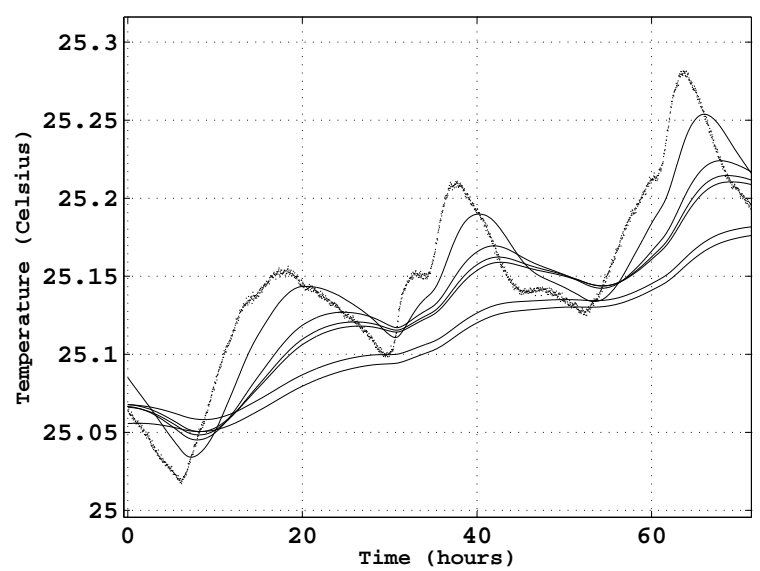

Fig. 8 Temperatures: $T(t)$ and $\psi_{j}(t)+\psi_{0, j}(t), j=1: 6$ (zoom)

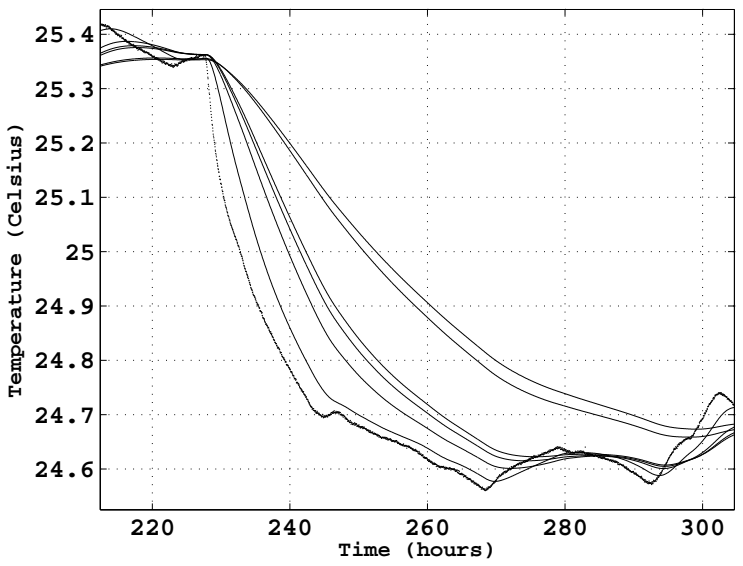

Fig. 9 Temperatures: $T(t)$ and $\psi_{j}(t)+\psi_{0, j}(t), j=1: 6$ (zoom)

C. Casenave, G. Montseny, Diffusive Identification of Volterra Models by Cancellation of the Nonlinear Term, 15th IFAC Symposium on System Identification, SYSID 2009, Saint-Malo (France), pp 640-645, July 6-8, 2009.

C. Casenave, E. Montseny, H. Camon, Identification of Nonlinear Dynamic Models of Electrostatically Actuated MEMS, Control Engineering Practice, Vol.18, N. 8, pp.954-969, 2010.

C. Casenave, G. Montseny, Identification of Nonlinear Volterra Models by means of Diffusive Representation, 17th IFAC World Congress, Seoul (Korea), pp.4024-4029, July 6-11, 2008.

C. Casenave, G. Montseny, Introduction do diffusive representation, To 4th IFAC Symposium on System, Structure and Control, SSSC 2010, Ancona (Italy), 2010.

A. Kärkkäinen, N. Pesonen, M. Suhonen, A. Oja, A. Manninen, N. Tisnek, H. Seppä, MEMS based AC 


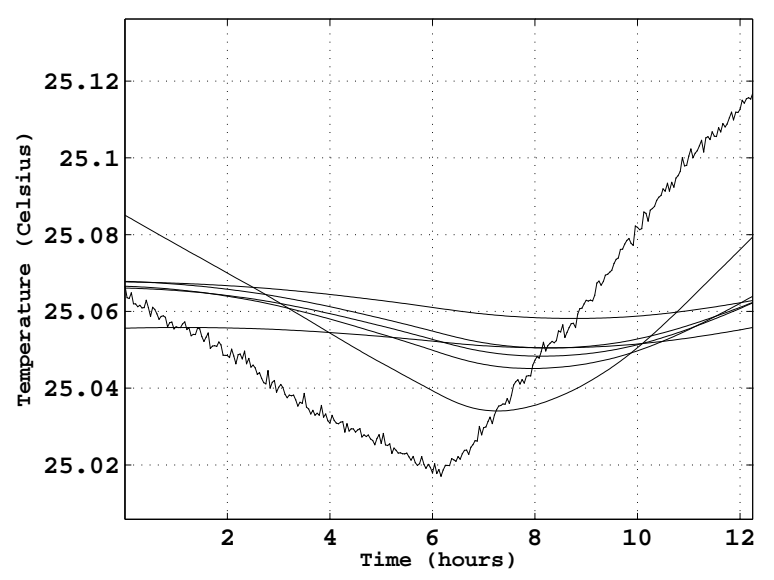

Fig. 10 Temperatures: $T(t)$ and $\psi_{j}(t)+\psi_{0, j}(t), j=1: 6$ (zoom)

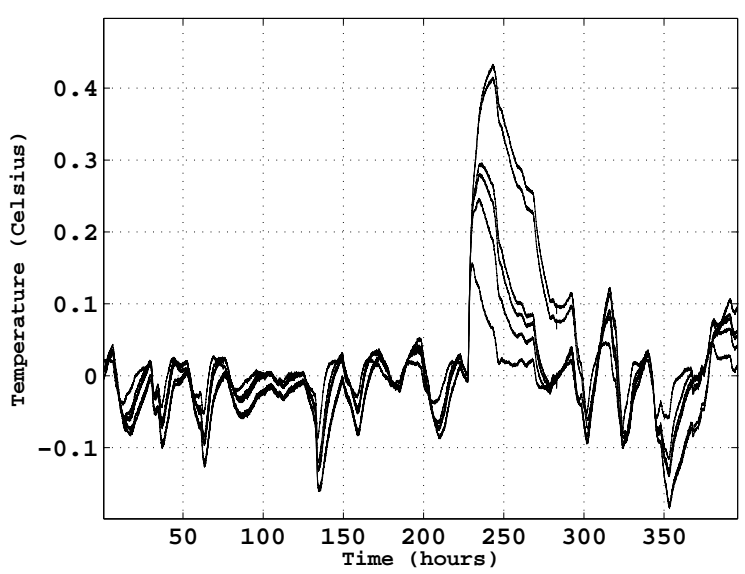

Fig. 11 Dynamic differences of temperatures: $\Psi_{j}(t)=\psi_{j}(t)+$ $\psi_{0, j}(t)-T(t), j=1: 6$

voltage reference, IEEE Trans. Instrum. Meas. 54, pp. 595-599, 2005

S. Krylov, B. R. Ilic, D. Schreiber, S. Seretensky, H. Craighead, The pull-in behavior of electrostatically actuated bistable microstructures, J. of Micromechanics and Microengineering, Vol. 18, 2008.

J. Kyynäräinen, A.S. Oja and H. Seppä, Stability of $m i$ croelectromechanical devices for electrical metrology, IEEE Trans. Instrum. Meas. 50, pp. 1499-1503, 2001.

G. Montseny, Représentation diffusive, Hermès Science, 2005.

M. Suhonen, H. Seppä, A.S. Oja, M. Heinilä, I. Näkki, $A C$ and $D C$ voltage standards based on silicon micromechanics, CPEM'98 Conf. Dig, pp. 23-24, 1998. 